\title{
Sustentabilidad de agroecosistemas de maíz de la planicie costera del
}

\section{Istmo, Oaxaca, México}

\author{
Rasgado Cabrera, Víctor Ernesto ${ }^{1,3}$; Ernesto Castañeda Hidalgo²; Salvador Lozano Trejo²; \\ María Isabel Pérez León ${ }^{2}$; Gisela Margarita Santiago Martínez ${ }^{2}$
}

1MCPA Instituto Tecnológico del Valle de Oaxaca (ITVO), México; ${ }^{2}$ DEPI-ITVO. Ex-Hacienda de Nazareno, Santa Cruz Xoxocotlán, Oaxaca, México; ${ }^{3}$ agrovic.vr@gmail.com

Rasgado Cabrera, Víctor Ernesto; Ernesto Castañeda Hidalgo; Salvador Lozano Trejo; María Isabel Pérez León; Gisela Margarita Santiago Martínez (2019) Sustentabilidad de agroecosistemas de maíz de la planicie costera del Istmo, Oaxaca, México. Rev. Fac. Agron. Vol 118 (2): 1-12. https://doi.org/10.24215/16699513e028

Los agroecosistemas tradicionales poseen diferentes características productivas, económicas, culturales y sociales; que provocan variabilidad del estado de sustentabilidad de cada uno de ellos. El objetivo fue evaluar niveles de sustentabilidad de los principales agroecosistemas de maíz en la planicie costera del Istmo de Tehuantepec; para diseñar futuras acciones de mejora. El estudio se realizó en el Istmo de Tehuantepec, Oaxaca, México en 2017. Se utilizó la metodología del MESMIS. Las técnicas de investigación fueron el cuestionario, recorridos de campo y diálogo con productores. Se diferenciaron tres agroecosistemas acorde al manejo y características propias. Se evaluaron 18 indicadores distribuidos en los atributos: productividad, confiabilidad, estabilidad, resiliencia, adaptabilidad, equidad y autogestión; que a su vez son trasversales a las dimensiones ambiental, social y económica. Al integrar los resultados por atributos, sobresale el $A E 1$ con un avance alto a la sustentabilidad (70\%), por mayor producción anual, eficiencia económica y más agricultores organizados. El AE2 alcanza un nivel bajo (47\%) debido al reducido ingreso mensual, actividades económicas limitadas, reducida superficie cultivable y nula capacitación y organización. El AE3 logra un nivel bajo (56\%), alcanzado por una baja diversidad de actividades económicas y mayor número de agricultores que reciben asesoría técnica. Al evaluar las dimensiones de la sustentabilidad, el AE1 y AE3 sobresalen con valores medios de avance hacia la sustentabilidad (74 y 63\%) y el AE2 muestra debilidad (53\%). Para mejorar estos niveles, se requieren diseñar y promover programas de desarrollo agrícola mediante procesos participativos que contemplen la innovación tecnológica con enfoque agroecológico.

Palabras clave: adaptabilidad, agricultura campesina, MESMIS, productividad, resiliencia

Rasgado Cabrera, Víctor Ernesto; Ernesto Castañeda Hidalgo; Salvador Lozano Trejo; María Isabel Pérez León; Gisela Margarita Santiago Martínez (2019) Maize agroecosystems sustainability in the coastal plain of Istmo, Oaxaca, Mexico.Rev. Fac. Agron. Vol 118 (2): 1-12. https://doi.org/10.24215/16699513e028

Traditional agroecosystems have different productive, economic, cultural and social characteristics; that cause variability of the sustainability status of each one of them. The objective was to evaluate sustainability levels of the main maize agroecosystems in the coastal plain of the Isthmus of Tehuantepec; to design future improvement actions. The study was carried out in the Isthmus of Tehuantepec, Oaxaca, Mexico in 2017. The MESMIS methodology was used. The research techniques were the questionnaire, field trips and dialogue with producers. Three agroecosystems were differentiated according to their own management and characteristics. 18 indicators distributed in the attributes were evaluated: productivity, reliability, stability, resilience, adaptability, equity and self-management; which in turn are transverse to the environmental, social and economic dimensions. By integrating the results by attributes, AE1 stands out with a high advance to sustainability $(70 \%)$, due to higher annual production, economic efficiency and more organized farmers. AE2 reaches a low level (47\%) due to reduced monthly income, limited economic activities, reduced arable land and no training and organization. AE3 achieves a low level (56\%), achieved by a low diversity of economic activities and a greater number of farmers who receive technical assistance. When evaluating the dimensions of sustainability, AE1 and AE3 stand out with medium values of progress towards sustainability (74 and 63\%) and AE2 shows debility (53\%). To improve these levels, it is necessary to design and promote agricultural development programs through participatory processes that contemplate technological innovation with an agroecological approach.

Keywords: adaptability, peasant agriculture, MESMIS, productivity, resilience

https://revistas.unlp.edu.ar/revagro

Recibido: $14 / 03 / 2019$

Aceptado: $13 / 09 / 2019$

Disponible on line: $27 / 12 / 2019$

ISSN 0041-8676 - ISSN (on line) 1669-9513, Facultad de Ciencias Agrarias y Forestales, UNLP, Argentina 


\section{INTRODUCCIÓN}

Desde el planteamiento del desarrollo sustentable en el Informe Brundtland en 1987, el concepto de sustentabilidad adquirió un papel relevante en las ciencias ambientales y cobró interés en las agencias nacionales debido a la importancia que representa para la humanidad (Kumar et al., 2009; Gómez et al., 2015). En el informe se puntualizó el concepto del desarrollo sustentable, entendido como aquel que puede satisfacer las necesidades de la generación actual, sin comprometer la capacidad de las generaciones venideras, para también se puedan satisfacer sus propias necesidades (WCED, 1987; MazabelDomínguez et al., 2010; Soflaei et al., 2016). Es decir, sin comprometer los sistemas de soporte vital del planeta y el bienestar de las generaciones futuras. Bajo este principio, el sector agrícola debe satisfacer las necesidades alimentarias de la población y simultáneamente, preservar los sistemas naturales que sustentan la vida (Valdez-Velazquez, 2017).

Para satisfacer las necesidades esenciales, el ser humano transforma los ecosistemas de su forma natural, haciéndolos interactuar con factores, principalmente tecnológicos, que los artificializan, lo que afecta las diferentes dimensiones de la sustentabilidad (Zagoya, 2015). Ante esto, Sarandón y Flores (2009) y Flores y Sarandón (2015) plantean la necesidad de un cambio hacia un modelo agrícola sustentable. Es decir, ecológicamente apropiado (ambientalmente sostenible), socialmente más justo y económicamente viable (Sánchez, 2003). En el contexto de la agricultura campesina, la sustentabilidad significa desarrollar y operar sistemas de manejo de los recursos naturales que sean productivos, estables, adaptables, confiables y resilientes, que distribuyan sus costos y beneficios de manera equitativa y generen procesos autogestivos y autónomos entre los beneficiarios (Astier, 2006).

Algunas de las estrategias comúnmente planteadas para mejorar la sustentabilidad de los $A E$, en especial la productividad y resiliencia se encuentran la diversificación de especies vegetales en el tiempo y espacio, la sustitución de fertilizantes sintéticos por el reciclaje de biomasa y enmiendas orgánicas para mejorar fertilidad del suelo, etc., las cuales inciden de manera positiva en la dimensión ambiental y económica (Altieri y Toledo, 2011; Altieri y Nicholls, 2012, 2013). Asimismo, con el propósito de subsanar ciertas carencias, es preciso diversificar las actividades en la unidad de producción familiar para aprovechar al máximo los bienes y servicios del capital natural (Pretty, 2008; Magdaleno-Hernández et al., 2014).

Para México, el maíz es un cultivo de gran importancia, pues garantiza la seguridad alimentaria de millones de familias rurales (Ortiz et al., 2010). Aproximadamente $92 \%$ de la superficie sembrada se localiza en zonas de agricultura de secano, dependientes de las lluvias de primavera y verano (Astier et al., 2003). Estos agroecosistemas (AE) son característicos de la agricultura campesina y han surgido a través de siglos de evolución biológica y cultural, se presume que tienden a ser sostenibles en materia ambiental, social y económica, ya que se aplican los principios agroecológicos. Sin embargo, no se sabe exactamente qué tan lejos o cerca se encuentran del objetivo de la sustentabilidad y cuáles son las razones de ésta (Astier, 2006). Por ello, los AE tradicionales se consideran escenarios óptimos para evaluar propiedades de sustentabilidad y para obtener criterios sobre el diseño y manejo de AE alternativos (Ruiz y Silva, 2006; Sarandón et al., 2008). Por lo tanto, es necesario analizar si realmente estos $\mathrm{AE}$ cumplen con el objetivo de la sustentabilidad, de tal manera que se puedan predecir problemas futuros y se brinden recomendaciones tendientes a su solución (Sarandón et al., 2008).

La medición o evaluación de la sustentabilidad, es una tarea desafiante debido la complejidad y multidimensión del concepto, por lo que para reflejar el estado general o grado de sustentabilidad de los $A E$ se requiere un abordaje sistémico que integre las distintas dimensiones (Sarandón et al., 2008; y Flores y Sarandón, 2015). En contraste con lo que sucede con los enfoques convencionales que se basan únicamente en la dimensión económica, lo que no basta para evaluar el funcionamiento a largo plazo de los $A E$ campesinos, los cuales son subvaluados cuando se utilizan únicamente criterios monetarios de corto plazo (Masera y López-Ridaura, 2000; Albicette et al., 2009). Por ello, es necesario el uso de enfoques metodológicos interdisciplinarios que empleen métodos prácticos para mostrar y explicar cómo interactúan las dimensiones social, económica y ambiental, que permitan la operación y desarrollo del sistema de producción (Castillo et al., 2012; Valdez-Vazquez et al., 2017).

El Marco para la Evaluación de Sistemas de Manejo de Recursos Naturales Incorporando Indicadores de Sustentabilidad (MESMIS) propuesto por Masera et al. (1999), permite operacionalizar el concepto de sustentabilidad de una manera multidimensional (Valdez-Velázquez, 2017). Debido a que no existe un conjunto de indicadores universales que puedan ser utilizados para diagnosticar el estado de un $A E$, es necesario construir indicadores acorde a la situación en estudio y los objetivos del mismo, que permitan conocer de manera particular sus cualidades (Altieri 1999; Sarandón et al., 2008). Los indicadores son variables que describen el estado de los $\mathrm{AE}$, generalmente a través de datos observados o estimados y advierten sobre la posición del $A E$ en cuestión, en relación con los límites u objetivos de la sustentabilidad propuestos (Astier y González, 2008). Estos indicadores deben mostrar valores claros, objetivos y generales, de carácter cualitativo y cuantitativo para favorecer un abordaje sistémico del tema y comprender perfectamente, sin ambigüedades, el estado de la sustentabilidad del AE; y como consecuencia, tomar decisiones para su mejora (Sarandón, 2002; Flores y Sarandón, 2015).

De acuerdo con Ramírez et al. (2015), la estrategia de reproducción social de las familias campesinas de Juchitán, Oaxaca se encuentra vinculada a la producción, comercialización y transformación de productos agrícolas, especialmente el maíz. En ese sentido, es importante el fomento de proyectos productivos orientados al fortalecimiento de la organización social campesina. Es fundamental fomentar el capital social a través del reconocimiento de la identidad cultural, los métodos participativos y las 
redes de agricultores para el desarrollo e intercambio de innovaciones en la solución de problemas (Altieri y Nicholls, 2007; 2010). Es importante considerar el uso de variedades locales mejoradas mediante el fitomejoramiento participativo y aumentar la agrobiodiversidad para lograr una mejor adaptación a las condiciones cambiantes del ambiente y exigencias del mercado (Martínez et al., 2006).

En la planicie costera del Istmo de Tehuantepec persisten $\mathrm{AE}$ o sistemas agrícolas campesinos, cuya variabilidad económica, social, productiva y cultural, hacen de estos territorios escenarios idóneos para realizar estudios de sustentabilidad, con el fin de preservar y mejorar las formas de manejo tradicionales y se contribuya a la sustentabilidad. Tal es el caso de las zonas de Juchitán-Chicapa de Castro, Álvaro Obregón-Emiliano Zapata y La Venta-La Ventosa. En cada una de estas zonas existen AE donde se produce maíz bajo diferentes procesos productivos; la evaluación de la sustentabilidad bajo estas condiciones, sustentan la presente investigación, que responde a uno de los problemas más acuciantes de la agricultura moderna, ya que trata la sustentabilidad como eje fundamental de la investigación.

Para estas zonas no existen estudios que demuestren el grado de sustentabilidad en el que se encuentran dichos $A E$, que de cierta manera, puedan servir de referencia para generar alternativas de manejo bajo principios agroecológicos. En este sentido, es necesario realizar un análisis integral de la dinámica actual de la agricultura local, por lo que el objetivo de esta investigación fue evaluar la sustentabilidad de tres $A E$ de maíz en la planicie costera del Istmo de Tehuantepec para el diseño de acciones de mejora.

\section{MATERIALES Y MÉTODOS}

\section{Descripción del área de estudio}

La investigación se realizó en el municipio de Juchitán de Zaragoza, el cual se ubica en la planicie costera del Istmo de Tehuantepec, Oaxaca, México. Este municipio cuenta con la mayor parte de las tierras irrigadas por el Distrito de Riego 19. Geográficamente se sitúa entre los paralelos $16^{\circ} 12^{\prime}$ y $16^{\circ} 38^{\prime} \mathrm{LN}$ y $94^{\circ} 44^{\prime}$ y $95^{\circ} 08^{\prime} \mathrm{LO}$, con una altitud media de 30 m (INEGI, 2010a; 2010b; UABJO, 2014). Comprende, además de la cabecera municipal, cinco agencias municipales y dos agencias de policía. El clima es un $A w_{0}(w)$ ig, catalogado como un clima cálido subhúmedo, el más seco de los subhúmedos con régimen de lluvias en verano; temperatura media de $26{ }^{\circ} \mathrm{C}$ y precipitación media anual de $978 \mathrm{~mm}$. En la zona de estudio predominan los suelos Vertisol, Phaeozem, Arenosol, Luvisol, Fluvisol, Cambisol, Solonchak y Gleysol (INEGI, 2010a; 2010b).

\section{Metodología de investigación}

La información de campo se generó a partir de un cuestionario, recorridos de campo y el diálogo directo y abierto con los productores. El cuestionario se estructuró en aspectos sociales, económicos, productivos y de organización de las unidades de producción campesinas. Para su aplicación se seleccionaron informantes claves o conocedores locales en cada una de las comunidades, consideradas como estratos, que fueron las localidades, bajo el criterio de que éstos fueran agricultores dedicados al cultivo de maíz. Para su selección se usó la técnica de la "bola de nieve"; la cual consiste en hacer el primer acercamiento con un informante clave o conocedor local, a quien se le aplica el cuestionario, posteriormente se le pide mencionar quién o quiénes podrían ser los próximos informantes clave. El tamaño de muestra se calculó usando el $5 \%$ de agricultores registrados en el padrón del Centro de Apoyo al Desarrollo Rural (CADER) No. 4 en las localidades en estudio. La muestra consistió en 60 agricultores de un total de 1,200.

Para la evaluación de la sustentabilidad se empleó el proceso metodológico MESMIS propuesto por Masera et al. (1999). La evaluación fue de carácter comparativo transversal y se basó en el análisis simultáneo de tres $\mathrm{AE}$ de maíz previamente identificados. Esta metodología propone seis pasos cíclicos:

1- Determinación del objeto de estudio. De acuerdo con la metodología, la sustentabilidad debe evaluarse en términos relativos; es decir, comparando dos o más situaciones. Por lo que el primer paso es definir y describir a los AE. Para el caso, se identificaron y caracterizaron tres $A E$ a partir de los aspectos biofísicos, tecnológicos, sociales y económicos: 1. Agroecosistema de maíz en Juchitán-Chicapa de Castro, 2. Agroecosistema de maíz en Álvaro ObregónEmiliano Zapata y 3. Agroecosistema de maíz en La Venta-La Ventosa.

2-Determinación de los puntos críticos del sistema (fortalezas y debilidades). Estos reflejan aspectos importantes que debilitan o fortalecen la sostenibilidad del sistema, deben relacionarse con los atributos adecuados.

3-Selección de los indicadores de sustentabilidad. Consiste en establecer criterios de diagnóstico vinculados a los atributos y puntos críticos. Posteriormente se construye una lista de indicadores potenciales, los cuales tienen unidades específicas para su evaluación.

4-Medición de los indicadores. Medición de los indicadores seleccionados previamente mediante el uso de técnicas como encuestas, cuestionarios, entrevista, mediciones en campo, entre otras.

5-Presentación e integración de resultados. Los resultados de la medición de los indicadores son sintetizados e integrados en diagramas tipo AMOEBA, los cuales muestran de manera instantánea el grado en que los valores de los sistemas de referencia $y$ alternativo se encuentran con respecto a los valores óptimos de los indicadores.

6-Conclusiones y recomendaciones. Partiendo del diagrama de AMOEBA, se discuten las diferentes características del sistema en términos de sostenibilidad $y$ se hacen conclusiones $y$ recomendaciones. A partir de las recomendaciones, comienza el primer paso de un nuevo ciclo de evaluación.

EI MESMIS se basa en la evaluación de siete atributos relevantes para la sustentabilidad: productividad, estabilidad, confiabilidad, resiliencia, adaptabilidad, equidad y autodependencia (Masera et al. 2000; Speelman, 2007). Por tal razón, en los $A E$ antes 
mencionados se identificaron la estructura, componentes, insumos, flujos internos y productos generados. Se evaluaron 18 indicadores cualitativos y cuantitativos distribuidos en los diferentes atributos de sustentabilidad propuestos por el MESMIS. Finalmente se sistematizó una base de datos para su análisis. El valor óptimo se basó principalmente en el máximo registrado para cada indicador, así como valores óptimos consultados en la literatura disponible. La ponderación de los valores fue con base en lo expuesto por Nahed (2008), quien indica que el valor óptimo es la cifra máxima.

Finalmente, se estableció la regla de decisión para conocer el nivel de sustentabilidad del AE, basada a partir del cálculo del porcentaje de sustentabilidad de cada atributo, el cual resultó del promedio de los porcentajes de cada indicador con respecto al valor óptimo para cada uno. Posteriormente, el porcentaje de sustentabilidad de cada AE se obtuvo a partir del porcentaje promedio de cada atributo. Finalmente el dato obtenido se comparó con el valor cualitativo dado a la sustentabilidad, valoración basada en las escala relativa de sustentabilidad (Marini, 2012) (Tabla 1).

Tabla 1. Escala relativa de sustentabilidad.

\begin{tabular}{lc}
\hline Escala relativa & Nivel de sustentabilidad \\
\hline $81-100$ & Alto \\
$61-80$ & Medio \\
$41-60$ & Bajo \\
$21-40$ & Potencialmente insustentable \\
$0-20$ & Insustentable \\
\hline
\end{tabular}

\section{RESULTADOS}

\section{Determinación del objeto de estudio}

1-Características de los agroecosistemas

Se identificaron y caracterizaron tres $A E$ a partir de los aspectos biofísicos, tecnológicos, sociales y económicos: AE1. Agroecosistema de maíz en Juchitán-Chicapa de Castro, AE2. Agroecosistema de maíz en Álvaro Obregón-Emiliano Zapata y AE3. Agroecosistema de maíz en La Venta-La Ventosa (Tabla 2).

Se puede apreciar que las características biofísicas de los $\mathrm{AE}$ estudiados presentan similitudes en cuanto a temperaturas máximas y medias; sin embargo, las temperaturas mínimas son variables, lo que trae consigo variaciones importantes en la respuesta del cultivo en épocas de frio. De la misma manera, se aprecia que en el AE de Álvaro Obregón-Emiliano Zapata (AE2), persisten los suelos Phaeozem, que si bien son suelos ricos en materia orgánica producto de la descomposición y sedimentación de material vegetal, también presentan altos niveles de acumulación de carbonatos y otras sales propias de la altitud donde se ubica el AE. Por clima se cataloga como seco y árido; lo que limita el rendimiento del maíz. Es importante destacar que este tipo de suelos también se presenta en los $A E 1$ y $A E 3$. Sin embargo, en dichos $A E$ se encuentran otros agrupamientos de suelos, lo que permite diversificar el manejo y mejorar el rendimiento del maíz, en especial en el AE1, lo cual justifica una mejor respuesta productiva.

En los ámbitos social, económico y tecnológico, se destaca el grado de asociación en que solo se encuentran los productores del $A E 1$, los cuales presentan la mayor edad promedio por productor. Los integrantes de la familia que más participan en las actividades relacionadas con la producción del maíz son los del AE2; sin embargo, son los que menos ingresos reportan debido a los bajos niveles tecnológicos y la falta de asesoría técnica; factores que evidentemente limitan el rendimiento. Las pruebas multivariables de aproximación de $F$ muestran la diferenciación de los AE con una significancia menor de 0.001 y las máximas distancias cuadradas de Mahalanobis (Tabla 3).

Por otro lado, los agricultores de los tres AE pertenecen a la cultura zapoteca y tienen amplia preferencia por el cultivo de maíz nativo Zapalote Chico debido a su precocidad, adaptabilidad a las condiciones locales, resistencia a plagas y enfermedades y hábitos de consumo. Se observan similitudes en ciertas prácticas de conservación de suelos, como la incorporación de residuos de cosecha y de estiércol para mejorar la fertilidad y calidad del suelo. De igual manera, los agricultores reciben incentivos del programa PROAGRO Productivo del gobierno mexicano.

\section{2- Determinación de los puntos críticos}

Los puntos críticos seleccionados para la evaluación de los AE muestran una gran influencia sobre la eficiencia y la sostenibilidad de forma general, por tal razón impactan los ámbitos productivos, económicos y sociales (Tabla 4).

Posteriormente, en cada punto crítico se derivaron indicadores de respuesta mediante la participación y consenso con las comunidades operadoras de los $\mathrm{AE}$, lo cual hace pertinente, medible y socialmente justificado cada uno de ellos.

\section{3- Selección de los indicadores}

Si bien es cierto que se realizó una amplia propuesta de indicadores (103) para atender los diferentes puntos críticos como resultado del diagnóstico; también hay que reconocer que no todos podían resultar evaluados con eficacia y ser de relevancia para las comunidades campesinas involucradas en el estudio, por lo que para reducir la dimensionalidad se aplicó un Análisis de Componentes Principales (ACP) (SAS). Con este análisis fue posible seleccionar los indicadores de mayor peso relativo; en este caso 10, que permitieran evaluar las dimensiones económica, social y ambiental. El segundo criterio para seleccionar otra serie de indicadores fue el "juicio de valor" de acuerdo a Masera et al. (2000). Con el cual se incorporaron 8 indicadores más, catalogados como importantes para la caracterización de los $\mathrm{AE}$, para un total de 18 indicadores. Se realizó un Análisis de Discriminantes Canónicos (ADC) con el procedimiento CANDISC (SAS) para detectar discrepancias entre localidades, de ahí que los resultados de la Tabla 4 sean de importancia en el análisis, ya que las soluciones adaptadas o propuestas estarán acorde a las necesidades más imperantes de los AE evaluados. 
Tabla 2. Caracterización de los agroecosistemas en estudio. AE1: Agroecosistema de maíz en Juchitán-Chicapa de Castro, AE2: Agroecosistema de maíz en Álvaro Obregón-Emiliano Zapata, AE3 Agroecosistema de maíz en La VentaLa Ventosa.

\begin{tabular}{|c|c|c|c|c|}
\hline & Característica & AE1 & AE2 & AE3 \\
\hline \multirow{5}{*}{$\frac{\frac{O}{0}}{\frac{0}{0}}$} & Temp. máxima $\left({ }^{\circ} \mathrm{C}\right)$ & 31,8 & 29,8 & 32,8 \\
\hline & Temp. mínima $\left({ }^{\circ} \mathrm{C}\right)$ & 17,6 & 13,3 & 22,3 \\
\hline & Temp. media anual $\left({ }^{\circ} \mathrm{C}\right)$ & 27 & 26 & 27,5 \\
\hline & Precipitación (mm) & 980 & 942 & 1000.7 \\
\hline & Altitud (msnm) & 22 & 6 & 38.8 \\
\hline \multirow{2}{*}{$\begin{array}{l}\overline{\frac{\pi}{U}} \\
\bar{\delta} \\
\infty\end{array}$} & $\begin{array}{l}\text { Integrantes de la familia que participan } \\
\text { en la UP }\end{array}$ & 1,7 & 3,37 & 2,4 \\
\hline & Organización para la producción & Grupo de trabajo 'Xhuba'huini' & \multicolumn{2}{|c|}{$\begin{array}{l}\text { No están asociados a algún grupo de } \\
\text { trabajo }\end{array}$} \\
\hline \multirow{2}{*}{ 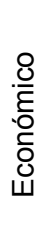 } & Monto de mano de obra (\$) & $\$ 170,00$ & $\$ 127,00$ & $\$ 150,00$ \\
\hline & Precio de venta del maíz $(\$ 1)$ & $\$ 7,00$ I & $\$ 6,80$ I & $\$ 7,801$ \\
\hline \multirow{9}{*}{ 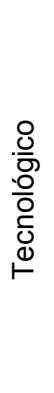 } & Método de labranza & Mixto & \multicolumn{2}{|r|}{ Mecanizada } \\
\hline & Método de siembra & Mixto & \multicolumn{2}{|r|}{ Mecanizada } \\
\hline & $\begin{array}{l}\text { Mano de obra empleada (jornales } \mathrm{ha}^{-1} \\
\mathrm{año}^{-1} \text { ) }\end{array}$ & 10 & 15 & 24 \\
\hline & Control de plagas & Químico & \multicolumn{2}{|r|}{ Ninguno } \\
\hline & Control de malezas & \multicolumn{3}{|c|}{ Solo en canales. Se aprovecha la biomasa como alimento para el ganado } \\
\hline & Superficie de siembra & 4,6 ha & 3,75 ha & 4,5 ha \\
\hline & No. de parcelas & 1,5 & 1,25 & 1,2 \\
\hline & Asesoría técnica & Si $(13,6 \%)$ & No & Si $(27,7 \%)$ \\
\hline & $\begin{array}{l}\text { Rendimiento acumulado } \\
\left(\mathrm{kg} \mathrm{ha}^{-1} \mathrm{año}^{-1}\right)\end{array}$ & 3,665 & 3,312 & 3,244 \\
\hline
\end{tabular}

Este tipo de trabajo permite la adopción y aceptación de las posibles soluciones que deriven es este análisis consensuado, ya que pocas veces se toman medidas o alternativas de solución frente a diversos problemas en las comunidades y no resultan efectivas, no son adaptadas por los campesinos y agricultores y lleva consigo el gasto de materiales, la ineficiencia económica y productiva. De hecho, este tipo de situaciones es tan frecuente que los gobiernos en todo mundo gastan millones de dólares en realizar cambios positivos en el campo y no se logran los resultados esperados, o no se sostienen en el tiempo, México no escapa de esta realidad.

\section{4- Medición de los indicadores}

La información para medir los indicadores se obtuvo a partir de información primaria generada en los cuestionarios y en la observación. Asimismo, se empleó información secundaria para la medición de ciertos indicadores. Se utilizó la evaluación mediante la estadística no paramétrica, a través de medidas de tenencia central.
5- Presentación e integración de resultados

Para una mejor visualización y comprensión de los resultados se utilizaron gráficas tipo AMOEBA y se segregó la integración de los resultados en indicadores, atributos y dimensiones (representados por cada eje en la gráfica). Los valores que se muestran en la figura 1 representan las mediciones obtenidas en el paso 4 . En este evaluación los indicadores óptimos se construyeron a partir de trabajos anteriores realizados en el área de estudio, así como el criterio del grupo evaluador.

Tabla 3. Distancia cuadrada de Mahalanobis

\begin{tabular}{cccc}
\hline Grupos & AE1 & AE2 & AE3 \\
\hline AE1 & 0 & 1138192214 & 101243517 \\
AE2 & 1138192214 & 0 & 1810314796 \\
AE3 & 101243517 & 1810314796 & 0 \\
\hline
\end{tabular}


Tabla 4. Criterios de diagnóstico, puntos críticos e indicadores por atributo de evaluación. A: Ambiental, S: Social, E: Económica.

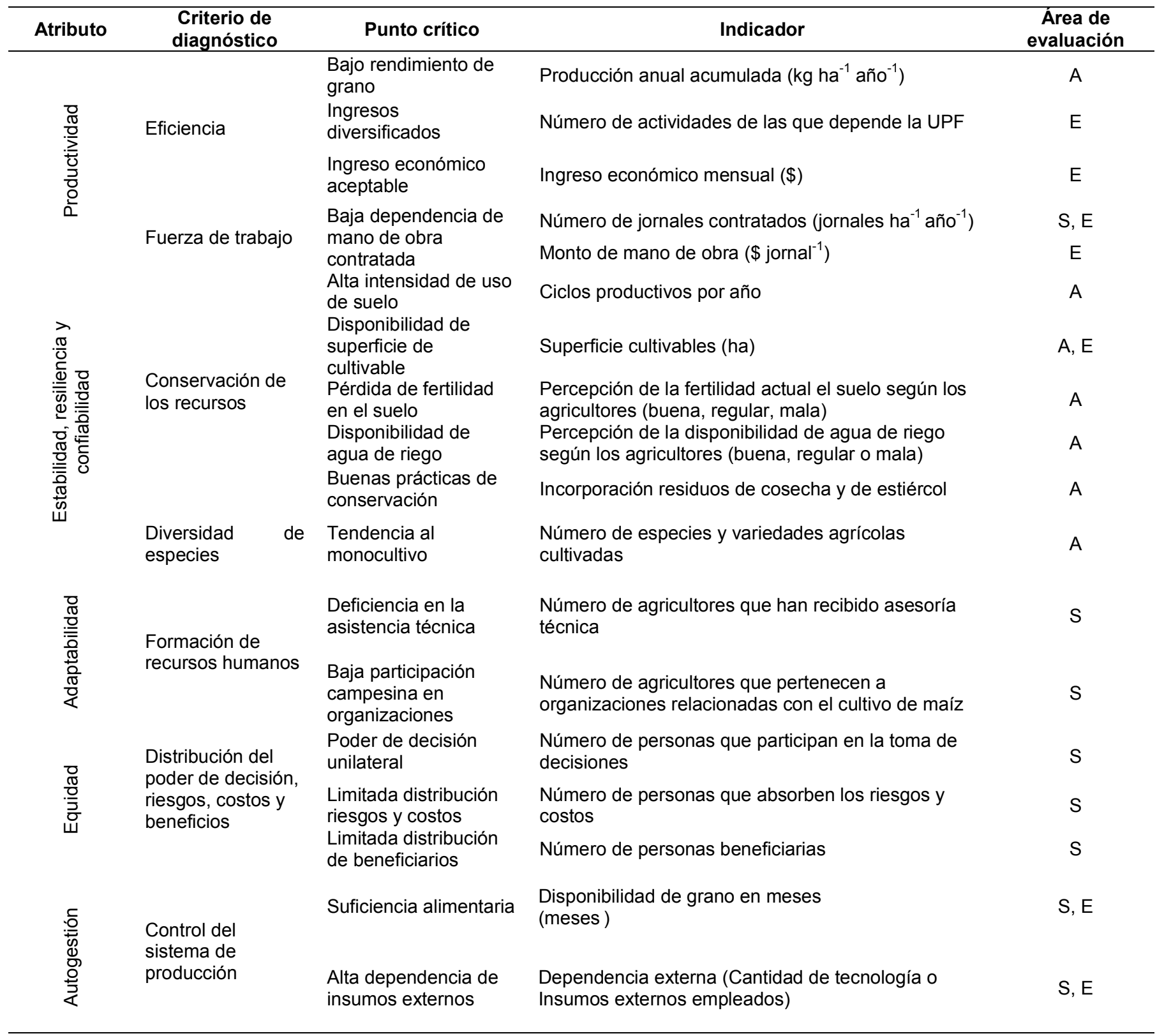

Visiblemente el AE2 obtuvo los peores resultados de forma general, solo la percepción de la fertilidad del suelo por parte de los productores fue evaluada con mucha mejor respuesta que los AE1 y AE3. Lo cual llama grandemente la atención, ya que la repercusión de la asistencia técnica en este $\mathrm{AE}$ tiene el peor comportamiento. Esto indica que el conocimiento local que se tiene sobre el nivel de la fertilidad del suelo es transmitido de generación en generación y forma parte de la cultura de trabajo dentro de este $A E$. Sin embargo, este conocimiento no se revierte en resultados productivos, obviamente porque existen otros factores, económicos y sociales, que influyen en la actividad y que resultaron con respuestas muy desfavorables para el AE2, que de manera general presenta las peores condiciones económicas, productivas y sociales.

\section{Evaluación por atributos}

Los resultados fueron homogenizados para su gráfica radial tipo AMOEBA (Figura 2).

1-Productividad. El AE1 sobresale al alcanzar un valor de $76 \%$, explicado por un mayor volumen de producción anual acumulada $\left(\mathrm{kg} \mathrm{ha}^{-1} \mathrm{año}^{-1}\right)$, por tener mayor ingreso económico mensual, número de jornales contratados anualmente y el monto de mano de obra $(\$$ jor $\left.^{-1}\right)$. 


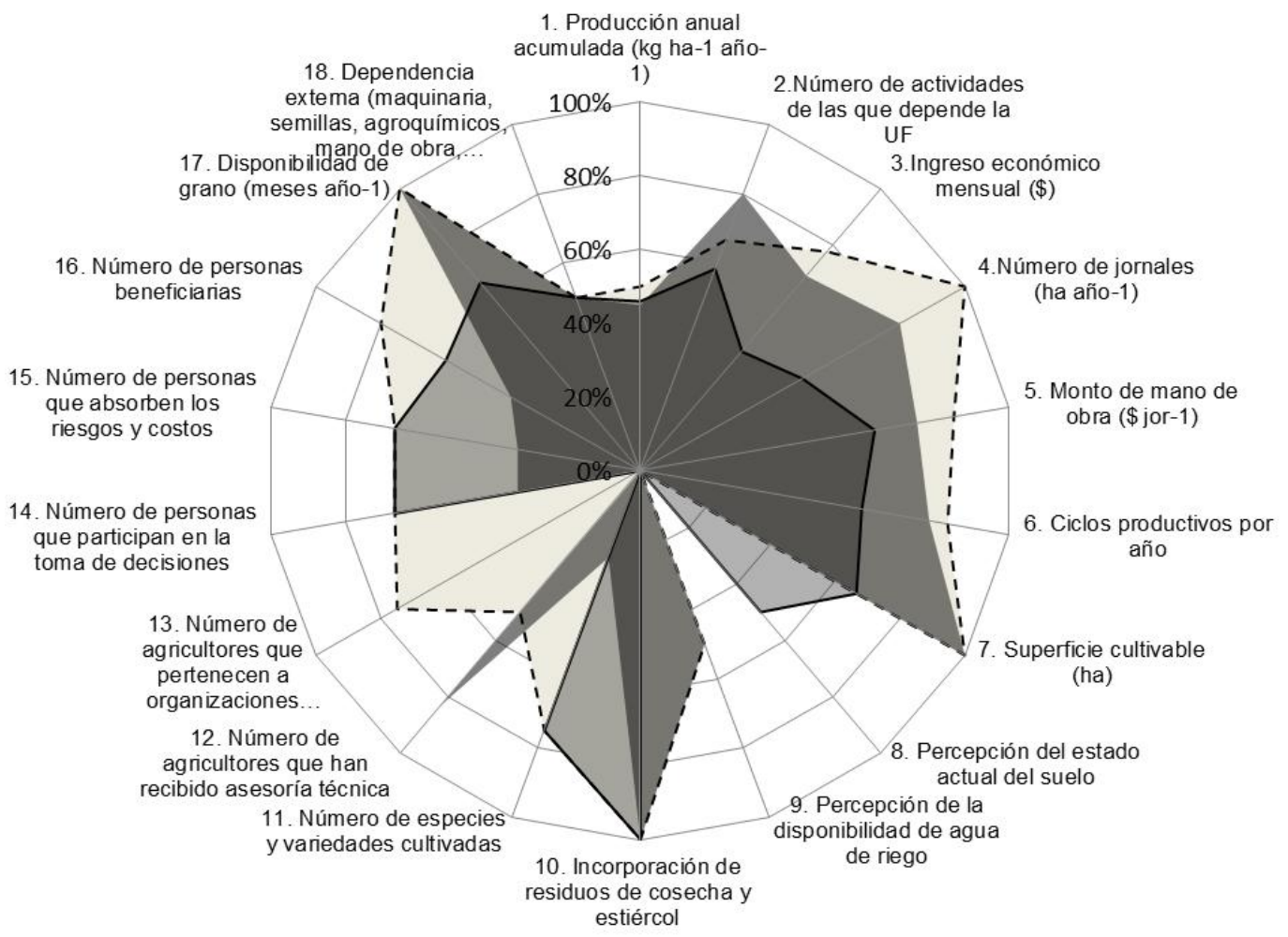

にAE1: $71 \%$

口AE2: $51 \%$

-AE3: $58 \%$

Figura 1. Integración de resultados por indicadores.

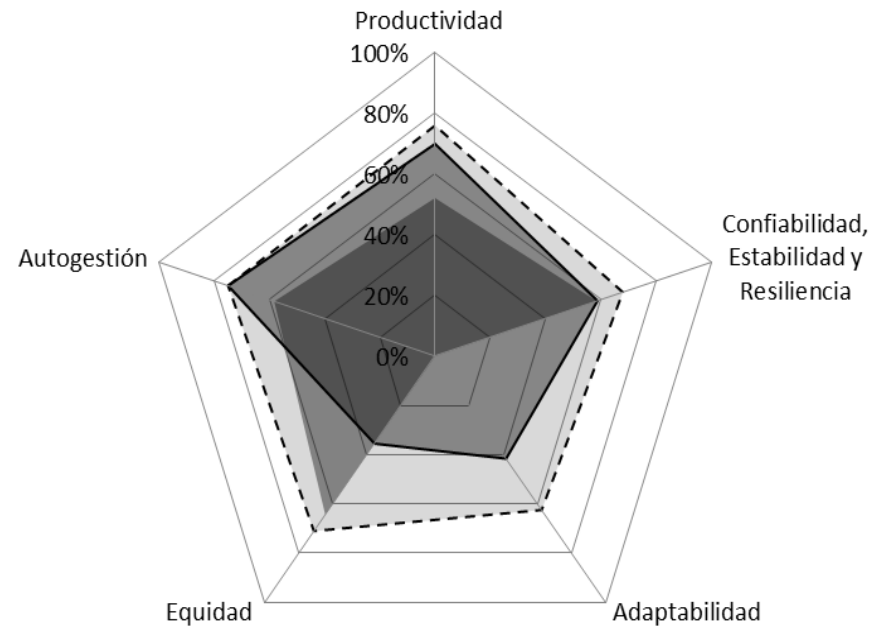

П. AE1: $70 \%$

AE2: $47 \%$

口AE3: $56 \%$

Figura 2. Integración de resultados por atributos de sustentabilidad. 
En contraste, el AE3 obtuvo un $70 \%$, caracterizado por una mayor diversidad de actividades económicas, aunque sus ingresos económicos son medios.

2-Confiabilidad, estabilidad y resiliencia. Sobresale el AE1 con un valor de $68,05 \%$, debido a una mayor superficie de tierra cultivable (ha) y más ciclos productivos por año; también por incorporar residuos de cosecha y estiércol y por conservar una mayor diversidad de especies cultivadas. EI AE2 alcanza un valor de $59 \%$ y se destaca por la calidad del suelo, que de acuerdo a la percepción de los agricultores no se ha deteriorado.

3-Adaptabilidad. Resalta el AE1 con un 63\%, explicado porque concentra el mayor número de agricultores que pertenecen a organizaciones o grupos de trabajo relacionados con el cultivo del maíz y asistencia técnica recibida. Por otra parte, el AE3 alcanza un 41,65\%, por un mayor número de agricultores que han recibido asesoría técnica. En contraste, el AE2 carece de asistencia técnica.

4-Equidad. El AE1 y AE2 presentan los mayores valores de equidad con 71,07 y 64,4\%, respectivamente, ya que integran el mayor número de personas que participan en la toma de decisiones, que absorben los riesgos y costos y que son beneficiarias. En contraste, el AE3 alcanza un promedio de $50 \%$ al concentrar menores valores para estos tres indicadores.

5-Autogestión. Sobresalen el AE1 y AE3 con un valor de $75 \%$, debido principalmente a la disposición de granos para la alimentación durante todo el año. En contraste, el AE2 alcanza un valor de $58,3 \%$, ya que los granos solo alcanzan para satisfacer las necesidades de las familias por ocho meses. En general, los tres $A E$ tienen una dependencia media de insumos externos.

De manera integral, al realizar la unificación de los resultados por atributos de acuerdo a la superficie coloreada y que representa a cada $\mathrm{AE}$, sobresale el AE1 con un nivel medio de avance hacia la sustentabilidad (70\%); y los AE3 y AE2 con un nivel bajo de contribución hacia la sustentabilidad (56 y 47\%, respectivamente).

\section{Evaluación por dimensiones}

Se realizó el análisis de acuerdo a las áreas o dimensiones de la sustentabilidad (ambiental, social y económica) (Figura 3).

En la dimensión ambiental se integraron siete indicadores; ocho en la social y siete en la económica. Sobresale el $A E 1$ al presentar el mayor valor (82,74\%), seguido del $A 3$ y del AE2 de menores valores (79 y $56,74 \%$, respectivamente). En cuanto a la dimensión social, destaca el AE1 (74\%) seguido del AE3 y del AE2 (52 y $45 \%$, respectivamente). Finalmente, en la dimensión económica sobresale el AE1 con el mayor valor (65\%), seguido del AE3 y del AE2 (ambos con $57 \%)$. En un contexto integral, el $A E 1$ tiene un nivel medio de avance hacia la sustentabilidad (74\%); sin embargo, se presenta débil en la dimensión económica. A pesar de tener ingresos contrastantes, el valor es bajo con respecto al promedio municipal reportado por el CONEVAL (2017), el cual es de $\$ 8,692$ para hogares con cuatro integrantes en promedio.

EI AE2 posee un nivel bajo de avance hacia la sustentabilidad (53\%), expresado en sus tres dimensiones; refleja menos actividades económicas y, como consecuencia, menor ingreso económico mensual; la superficie cultivable disponible es reducida, la producción anual de granos es mínima, por lo que el abasto de granos es insuficiente, además, se detecta la falta de asistencia técnica y la nula organización.

Finalmente, el AE3 alcanza un nivel medio de avance hacia la sustentabilidad (63\%). En su dimensión económica presenta como fortaleza el mayor número de actividades económicas realizadas, aunque el ingreso mensual no es el mayor. Además en este AE, los agricultores tienen mayor superficie cultivable $y$ disponibilidad de grano durante todo el año.

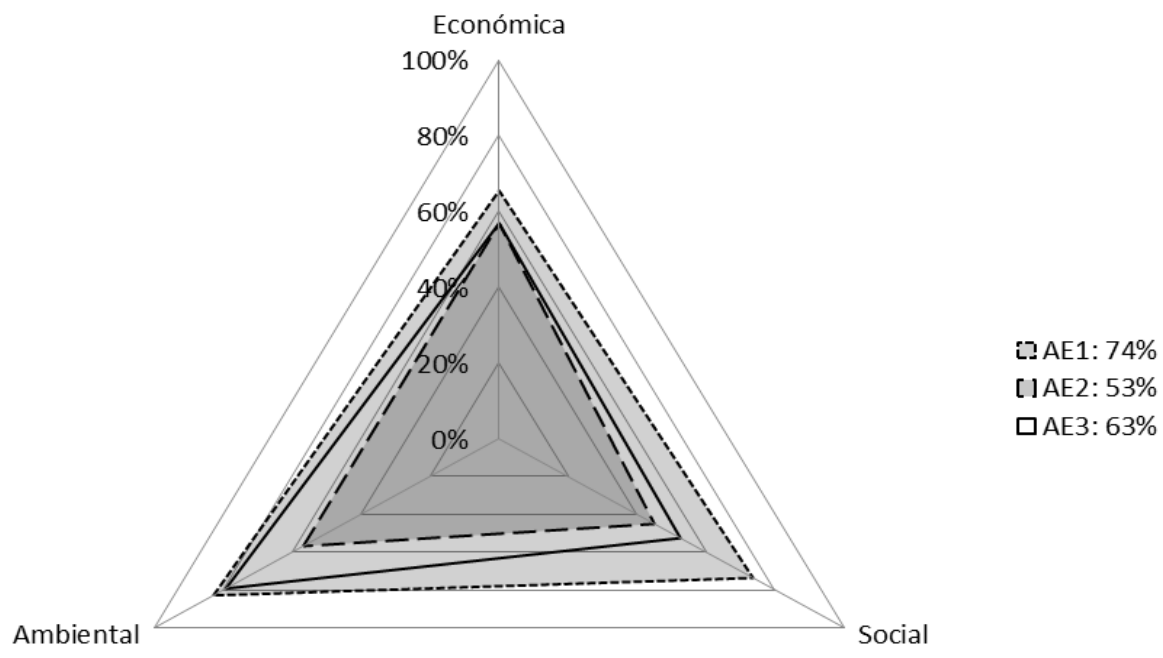

Figura 3. Sustentabilidad relativa integrando las dimensiones de evaluación. 


\section{DISCUSIÓN}

\section{La sustentabilidad en agroecosistemas}

La sustentabilidad y sus mecanismos de evaluación se han convertido en un proceso de gran utilidad que proporciona las herramientas básicas para evaluar a los $\mathrm{AE}$ con propósitos de superar las afectaciones a los aspectos ecológico-ambientales de la crisis de la agricultura moderna que implica aspectos económicos, productivos, sociales y culturales (Altieri, 1999). Por tal razón, este tipo de estudio se ha convertido en una nueva forma de hacer una ciencia más enfocada a las diversas problemáticas que pueden encontrarse en $\mathrm{AE}$ concretos, donde se evalúan indicadores pertinentes a cada situación y en estrecha relación con el factor humano, que a fin de cuentas es quien adoptara las medidas derivadas de su análisis (Gliessman, 2000).

En este sentido, los indicadores propuestos partieron de la situación concreta en términos productivos, sociales y económicos que existían en las comunidades, también en correspondencia con acciones afines al manejo agronómico del maíz, para orientar hacia la sustentabilidad a los $A E$ evaluados, para de esta manera contribuir con la calidad de vida de los habitantes y productores. Según Harold et al. (2006) se considera la construcción, utilidad y uso de indicadores concretos para evaluar la sostenibilidad de los sistemas productivos, es un avance metodológico y un instrumento de vital importancia de los cuales hace referencia el Instituto Interamericano de Cooperación para la Agricultura (IICA), por la estrecha relación que tienen los mismos con la evaluación del desarrollo sostenible de la agricultura y del medio rural; sin embargo, hay que destacar que cada $A E$ presenta sus propios indicadores de mayor impacto y depende de trabajo mancomunado entre los diferentes actores del proceso elegir los más adecuados (Sepúlveda et al., 2002). Tal y como se realizó en el presente estudio.

\section{Evaluación por atributos}

La evaluación de la sustentabilidad por atributos es de vital importancia, dado que estos atributos caracterizan el estado de los $A E$, por tal razón, la evaluación de la productividad, la confiabilidad, la estabilidad, la resiliencia, la adaptabilidad, la equidad y la autogestión, conforman el grupo de atributos establecidos para el estudio detallado de la sustentabilidad. En este sentido, Giraldo-Díaz et al. (2000) realizaron la evaluación de atributos de sustentabilidad de sistemas de producción campesinos en La Vereda El Mesón, municipio de Palmira, Valle del Cauca (Colombia), donde encontraron resultados similares a los obtenidos en este estudio. Los atributos evaluados mantuvieron resultados diferentes en cada uno de los $A E$ evaluados; sin embargo, la productividad y la autogestión resultaron puntos claves a tratar en el proceso de sustentabilidad.

Por otra parte, en este estudio, la respuesta obtenida en cada uno de los atributos es el reflejo de la poca aplicación de técnicas agroecológicas por parte de los campesinos para mantener la sustentabilidad, todo ello provocado pero el auge de la agricultura moderna y la promoción del uso de agroquímicos, mecanización, alta energía, etc., promovida por las políticas agrarias internacionales e implementadas a escala local, que en el causar de los años fuera cuestionada por los desequilibrios ecológicos que la misma ha provocado, con especial énfasis a las plantaciones o siembras especializadas, ajenas a las lógicas reproductivas propias de comunidades tradicionales, como el caso del maíz (Delgado et al., 2007).

También es importante destacar que la diversidad de resultados obtenidos en cada uno de los $A E$ en los diferentes atributos es lógica; autores como Rodríguez y Rodríguez (2010), han señalado que las comunidades, productores y $\mathrm{AE}$ nunca constituyen un conjunto homogéneo, porque que las diferencias edafoclimáticas, culturales, disponibilidad de recursos, organización, entre otros factores; no se comportan de la misma manera en todos los escenarios, de ahí la importancia de caracterizar los $\mathrm{AE}$ con este tipo de herramientas para que contribuya a una mejor toma de decisiones.

\section{Evaluación por dimensiones}

La evaluación por dimensiones también presentó diversidad debido a las características propias de cada $\mathrm{AE}$ y engloba de una manera muy rápida los principales problemas que pueden encontrarse y que afectan la sustentabilidad (Castañeda, 2005). Los resultados obtenidos muestran que los $A E$ presentan su principal debilidad en la dimensión económica, influenciada por diversos factores que intervienen este proceso, y que sin lugar a dudas es fundamental para lograr la sustentabilidad.

La dimensión económica demanda un desarrollo económicamente eficiente y equitativo dentro los $\mathrm{AE}$, para lograrlo se hace necesaria la utilización de recursos financieros, técnicos y humanos para desarrollar tecnologías amigables y con menor impacto en la dimensión ambiental y que permitan conservar y mejorar los rendimientos, muchas de ellas reconocidas como agroecológicas y que están centradas en el uso de abonos orgánicos, biofertilizantes, bajo uso de energía, etc. Esto debido a que el desarrollo económico desde la perspectiva de la sustentabilidad no puede basarse en la viabilidad de un proyecto, como ha sido el modo de actuación que se ha implementado en los últimos años, por lo que hay que considerar el impacto social y ambiental, para de una forma integral abordar las problemáticas que enfrentan los $\mathrm{AE}$ (Kammerbauer, 2001).

De igual manera, la dimensión social está dominada por la falta de asistencia técnica y capacitación, los cuales se consideran importantes (Damián et al., 2008; AyalaGaray et al., 2013); ya que refuerzan las habilidades y capacidades de los agricultores para enfrentar retos de mayor productividad, estabilidad y resiliencia ante los cambios locales provocados por el cambio climático y variaciones del mercado. Se deben diseñar y promover programas de desarrollo agrícola pero no bajo el esquema paternalista o asistencial. Lo apropiado es una combinación de elementos participativos entre los agricultores (Casas-Cázeres et al., 2009).

Asimismo, se debe fomentar la diversificación de los sistemas agrícolas. Mediante el mantenimiento de la diversidad del $A E$, se fortalece la productividad, adaptabilidad y resiliencia. Con estas prácticas es posible aumentar los rendimientos de maíz e 
incrementar la cantidad destinada a la venta, lo que se reflejaría en el aumento del nivel de ingreso económico.

\section{CONCLUSIONES}

La evaluación de la sustentabilidad de los sistemas agrícolas es de gran importancia para conocer el estado en que se encuentran los $A E$ y el impacto de las formas de manejo en los atributos y dimensiones. Mediante la aplicación de la metodología del MESMIS es posible identificar, de manera sistémica, aquellos aspectos que brindan fortaleza o debilitan a los $A E$, por lo que estudios de este tipo resultan de importancia para mejorar de toma de decisiones por parte de los productores, caso de maíz en Juchitán, Oaxaca.

En cuanto a la evaluación de los $A E$ por dimensiones, el $A E 1$ obtuvo los mejores valores en las dimensiones ambiental, social y económico; 82,74, 74 y $65 \%$, respectivamente; con un valor promedio total de $74 \%$. EI AE2 obtuvo los valores más bajos respecto a los otros dos $A E$ en las dimensiones ambiental, social y económica, con $56,74,45$ y $57 \%$, respectivamente; y un valor total promedio de $53 \%$. Por último, el AE3 obtuvo valores intermedios de 79,52 y $57 \%$ en las dimensiones ambiental, social y económica, y un valor total promedio de $63 \%$ de orientación hacia la sustentabilidad. Al comparar los valores totales y por dimensión entre los $A E$, las diferencias encontradas entre cada uno de ellos es de aproximadamente de un $10 \%$, con un orden de importancia $A E 1, A E 3$ y $A E 2$. Aunque el AE1 obtiene sus menores valores en el eje económico y los $A E 2$ y $A E 3$ en el eje social y con similitud de valores en la dimensión económico.

Respecto al valor total de los atributos, el AE1 alcanzó los mayores valores para un total de $70 \%$ de orientación hacia la sustentabilidad; alcanzando el valor más bajo de $63 \%$ para la adaptabilidad y el más alto, $76 \%$, para la productividad. EI AE2 obtuvo el valor más bajo, $47 \%$ de orientación hacia la sustentabilidad, con el menor valor en el atributo de adaptabilidad $(0 \%)$ y el más alto en el atributo de equidad $(64,4 \%)$. Mientras que el AE3 obtuvo un valor total de orientación hacia la sustentabilidad de $56 \%$ con el valor más bajo en el atributo de equidad $(35,53 \%)$ y el valor más alto en el atributo de autogestión (75\%).

Esto indica que el proceso para lograr la sustentabilidad requiere esfuerzos diferenciados para la mejora de cada uno de los indicadores en cada $A E$. En conjunto, el eje social es el de menor valor total general (57\%) y el que mayor diferencia establece entre el mejor y peor $A E: A E 1$ y $A E 2$, respectivamente; con valores de 74 y $45 \%$, lo que marca una diferencia de $29 \%$. Por lo que se requeriría atender de manera sustantiva este eje, en especial en los AE2 y AE3. El eje económico también tiene un valor bajo; sin embargo, no existen discrepancias tan marcadas entre los $\mathrm{AE}$; pero que en general, requieren de atención para mejorar la sustentabilidad. Por su parte, el eje ambiental es el mejor evaluado en los $\mathrm{AE}$; existiendo mucha similitud entre el $A E 1$ y $A E 3$; y muy superiores al AE2.

A manera de recomendación se sugiere orientar el uso de recursos económicos a la implementación de prácticas agroecológicas, las cuales son de bajo costo y sin impacto en el medio ambiente, reforzar la capacitación para el desarrollo de habilidades y capacidades y diversificar los sistemas agrícolas locales.

\section{BIBLIOGRAFÍA}

Albicette, M., R. Brasesco \& M. Chiappe. 2009. Propuesta de indicadores para evaluar la sustentabilidad predial en agroecosistemas agrícolaganaderos del litoral del Uruguay. Agrociencia. 8 (1): 48-68.

Altieri, M.A. 1999. Agroecología. Bases agroecológicas para una agricultura sustentable. Editorial NordanComunidad. Montevideo, Uruguay. 325 p

Altieri, M.A. \& C.I. Nicholls. 2007. Conversión agroecológica de sistemas convencionales de producción, teoría, estrategias y valuación. Ecosistemas. 16 (1): 3-12.

Altieri, M.A. \& C.I. Nicholls. 2010. Diseños agroecológicos para incrementar la biodiversidad de entomofauna benéfica en agroecosistemas. Sociedad Científica Latinoamericana de Agroecología. Medellín Colombia. 83 p.

Altieri, M.A. \& C.I. Nicholls. 2012. Agroecología: Única esperanza para la soberanía alimentaria y la resiliencia socioecológica. Sociedad Científica Latinoamericana de Agroecología. $21 \mathrm{p}$.

Altieri, M.A. \& C.I. Nicholls. 2013. Agroecología y resiliencia al cambio climático: principios y consideraciones metodológicas. Agroecología 8 (1): 7 20.

Altieri, M. \& V.M. Toledo. 2011. The agroecological revolution: Rescuing nature, ensuring food sovereignty and empowering peasants. The Journal of Peasant Studies. 38 (3): 587-612.

Astier, M. 2006. Medición de la sustentabilidad en sistemas agroecológicos. Memoria del VII Congreso de la Sociedad Española de Agroecología. Ponencia 3. México, D.F.: MundiPrensa-GIRA-ILEIA-ICCO. pp. 1-7. Astier, M. \& C. González. 2008. Formulación de indicadores socioambientales para evaluaciones de sustentabilidad de sistemas de manejo complejos. Capítulo 5. In: M. Astier, O. R. Masera \& Y. GalvánMyoshi (Cords). Evaluación de sustentabilidad. Un enfoque dinámico y multidimensional. SEAE, CIGA, ECOSUR, CIECO, UNAM. Mundiprensa. España. pp. 73-94.

Astier, M., E. Pérez-Agis, T. Ortiz \& F. Mota. 2003. Sustentabilidad de sistemas campesinos de maíz después de cinco años: el segundo ciclo de evaluación MESMIS. LEISA Revista de Agroecología.

Ayala-Garay, A.V., R. Schwentesius-Rindermann, P. Preciado-Rangel, G. Almaguer-Vargas \& P. RivasValencia. 2013. Análisis de rentabilidad de la producción de maíz en la región de Tulancingo, Hidalgo, México. Agricultura, sociedad y desarrollo. 10 (4): 381-395.

Cabrera, T.J.M., C.A. Carballo \& F. C. Aragón. 2015. Evaluación agronómica de maíces raza Zapalote Chico en la región istmeña de Oaxaca. Revista Mexicana de Ciencias Agrícolas. Pub. Esp. Núm. 11: 2075-2082.

Casas-Cázares, R., F.V. Gonzáles-Cossío, T. Martínez-Saldaña, E. García-Moya \& B.V. PeñaOlvera. 2009. Sostenibilidad y estrategia en 
agroecosistemas campesinos de los Valles Centrales de Oaxaca. Agrociencia. 43 (3): 319-331.

Castañeda, H. E. 2005. Metodología para el diagnóstico de agroecosistemas y evaluación de la sustentabilidad en Cuilapam de Guerrero, Oaxaca. Tesis Doctoral. Colegio de Postgraduados, Campus Puebla, Puebla, México.

Castillo R.D., M. Tapia R., L. Brunett P., O. Márquez M., O. Terán V. \& E. Espinosa A. 2012. Evaluación de la sustentabilidad social, económica y productiva de dos agroecosistemas de producción de leche a pequeña escala en el municipio de Amecameca, México. Revista Científica UDO Agrícola. 12 (3): 690-704.

Consejo Nacional de Evaluación de la Política de Desarrollo Social. (CONEVAL). 2017. Pobreza a nivel municipio 2010. http://www.coneval.org.mx/Medicion /MPI Paginas/Medicion-de-la-pobreza-municipal2010.aspx (Consultado: 20/06/2017)

Damián, H.M.Á., J.F. López O., B. Ramírez V., F. Parra I., J.A. Paredes S., A. Gil M. \& A. Cruz L. 2008. Hombres y mujeres en la producción de maíz: un estudio comparativo en Tlaxcala. Región y sociedad. 20 (42): 63-94.

Delgado, S.J., M.A. Fernández, G.E.M. Armenteros, L.O. Hernández \& P. Gavilanes. 2007. Análisis cualitativo y cuantitativo de la agricultura ecológica en Cuba. En: V.M.S. Garrido. Recomendaciones y estrategias para desarrollar la agricultura ecológica en Iberoamérica. Ed. CYTED. pp. 163-173.

Flores, C.C. \& S.J. Sarandón. 2015. Evaluación de la sustentabilidad de un proceso de transición agroecológica en sistemas de producción hortícolas familiares del Partido de La Plata, Buenos Aires, Argentina. Revista Facultad de. Agronomía. 114 (1): 52 66.

Giraldo-Díaz, R.; L.E. Nieto-Gómez \& A. QuicenoMartínez. 2000. Evaluación de atributos de sustentabilidad de sistemas de producción campesinos en la vereda El Mesón, municipio de Palmira, Valle del Cauca (Colombia). Revista Libre Empresa. 12(1), 111135. En: http://dx.doi.org/10.18041/libemp.v23n1.23106. 2015.

Gliessman, S. R. 2000. Agroecology: ecological process in sustainable agriculture. Ann Arbor Press Chelsea.

Gómez, L.F., L.A. Ríos-Osorio \& M.L. Eschenhagen D. 2015. El concepto de sostenibilidad en Agroecología. Rev. U.D.C.A Act. \& Div. Cient. 18(2): 329-337

Harold, A. M., G. X. Pedraza \& A.J. Solarte. 2006. Construcción y uso de indicadores de Sostenibilidad para la Planeación Participativa de Predios. Consultado [11-2006]. Disponible http://desarrollo.ecoportal.net/content/view/full/63022. Instituto Nacional de Estadística y Geografía (INEGI). 2010a. Anuario estadístico de Oaxaca edición 2010. En:http://www.inegi.org.mx/est/contenidos/espan ol/sistemas /aee10/info/oax/mapas.pdf. (Consultado: 01/02/2017).

Instituto Nacional de Estadística y Geografía (INEGI). 2010b. Compendio de información geográfica municipal 2010. Heroica Ciudad de Juchitán de Zaragoza. 9 p.

Kammerbauer, J. 2001. Las dimensiones de la sostenibilidad: fundamentos ecológicos, modelos paradigmáticos y senderos Interciencia. Asociación Interciencia. Caracas, Venezuela. 26(8): 353-359.

Kumar, S.R., H.R. Murty, S.K. Gupta, \& A.K. Dikshit. 2009. An overview of sustainability assessment methodologies. Ecological indicators. 9: 189-212.

Magdaleno-Hernández, E., M.A. Jiménez-Velásquez, T. Martínez-Saldaña \& B. Cruz-Galindo. 2014. Estrategias de las familias campesinas en Pueblo Nuevo, Municipio de Acambay, Estado de México. Agricultura Sociedad y Desarrollo. 11 (2): 167-179.

Marini, M.G. 2012. Evaluación de sustentabilidad en agroecosistemas de producción bovina en el Istmo, Oaxaca. Tesis de Maestría. Instituto Tecnológico del Valle de Oaxaca. 200 p.

Martínez, M., H. Ríos, S. Miranda, I. Moreno, R. Acosta, A. Farrera, \& J. Velasco. 2006. Caracterización de la diversidad y selección participativa de prospecciones de maíz en Chiapas, México. Cultivos Tropicales. 27 (1): 55-62.

Masera, O. \& S. López-Ridaura. 2000. Introducción. Cap. 1. In: Masera, O. \& López-Ridaura, S. (Eds.) Sustentabilidad y sistemas campesinos. Cinco experiencias en el México rural. Mundiprensa, Grupo Interdisciplinario de Tecnología Rural Apropiada A.C. UNAM. pp. 1-12

Masera, O., M. Astier \& S. López-Ridaura. 1999. Sustentabilidad y manejo de recursos naturales. El marco de evaluación MESMIS. Grupo Interdisciplinario de Tecnología Rural Apropiada A.C. Mundiprensa. 103 p.

Masera, O., M. Astier y S. López-Ridaura. 2000. El marco de evaluación MESMIS. Cap. 2. En: Masera, O. y López-Ridaura, S. (Eds.). Sustentabilidad y sistemas campesinos. Cinco experiencias en el México rural. Mundiprensa, Grupo Interdisciplinario de Tecnología Rural Apropiada A.C. UNAM. pp. 13-41.

Masera, O., M. Astier, S. López-Ridaura, Y. GalvánMiyoshi, T. Ortiz-Ávila, L.E. García-Barrios, R. García-Barrios, C. González \& E. Speelman. 2008. El proyecto de evaluación de sustentabilidad MESMIS. En: Evaluación de sustentabilidad. Un enfoque dinámico y multidimensional. M. Astier, O.R. Masera \& Y. GalvánMyoshi (coordinadores). SEAE, CIGA, ECOSUR, CIECO, UNAM. Ed. Mundiprensa. España. pp: 13-24.

Mazabel-Domínguez, D.G., M. Romero-Jacuinde \& M.A. Hurtado-Cardoso. 2010 La evaluación social de la sustentabilidad en la agricultura de riego. Ra Ximhai 6 (2): 199-219

Nahed, T.J. 2008. Aspectos metodológicos en la evaluación de la sostenibilidad de sistemas agrosilvopastoriles. Avances en Investigación Agropecuaria. 12 (3): 3-19

Nahmad S.S. 2011. El impacto social del uso del recurso eólico. Informe final para el Consejo Oaxaqueño de Ciencia y Tecnología (COCyT) del CONACYT. Proyecto 123396. 117 pp.

Negreros-Castillo, P., J.C. González N. \& L. Merino P. 2000. Evaluación de la sustentabilidad del sistema de manejo forestal de la Organización de Ejidos productores Forestales de la Zona Maya de Quintana Roo. En: Sustentabilidad y sistemas campesinos. Cinco experiencias de evaluación en el México rural. O. Masera, \& S. López-Ridaura (coordinadores). Grupo Interdisciplinario de Tecnología Rural Apropiada (GIRA A. C.). Mundi-Prensa. México. 83-142 p. 
Ortiz, R., S. Taba, V.H. Chávez T., M. Mezzalama, Y. Xu, J. Yan \& J.H. Crouch. 2010. Conserving and Enhancing Maize Genetic Resources as Global Public Goods-A Perspective from CIMMYT. Crop Science 50 (1): 13-28.

Pretty, J. 2008. Agricultural sustainability: concepts, principles and evidence. Phil. Trans. R. Soc. B. (363): 447.465.

Ramírez, M.C.A., L. Cruz A. \& V. Marcial C. 2015. Luchas por el territorio y la soberanía alimentaria en el Istmo oaxaqueño, México. Eutopía. (8): 29-44.

Rodríguez-Manzano, A. y Rodríguez-Rivera, A. 2010. Un guajiro científico. La Habana: Editorial CientíficoTécnica. ISBN: 978-958-05-0596-6.

Ruiz-Vega, J. \& M.E. Silva-Rivera. 2006. Caracterización de dos agroecosistemas presentes en suelos de ladera de la subcuenca Sur Oeste del Río Atoyac, Oaxaca. Naturaleza y Desarrollo 4 (2): 5-12.

Sánchez, C.M.C. 2003. Biotecnología: ventajas y desventajas para la agricultura. Revista Científica UDO Agrícola 3 (1): 1-11.

Sarandón, S. 2002. El desarrollo y uso de indicadores para evaluar la sustentabilidad de los agroecosistemas. En: Agroecología: El camino para una agricultura sustentable S. Sarandón (Ed.). Ediciones Científicas Americanas. La Plata, Argentina. pp. 393-414.

Sarandón, S.J. \& C.C. Flores. 2009. Evaluación de la sustentabilidad en agroecosistemas: una propuesta metodológica. Agroecología. (4): 19-28.

Sarandón, S.J., M.S. Zuluaga, R. Cieza, L. Janjetic \& E. Negrete. 2008. Evaluación de la sustentabilidad de sistemas agrícolas de fincas en Misiones, Argentina, mediante el uso de indicadores. Agroecología. (1): 1928.

Sepúlveda, S., H. Cavaría, A. Castro, P. Rojas, E. Picado \& D. Bolaños. 2002. Metodología para estimar el nivel de desarrollo sostenible en espacios territoriales. IICA. 47 p.

Soflaei, F., M. Shokouhian \& W. Zhu. 2016. Socioenvironmental sustainability in traditional courtyard houses of Iran and China. Renewable and Sustainable Energy Reviews 19: 1147-1169.

Speelman, E.N., S. López-Ridaura, N.A. Colomer, M. Astier \& O.R. Masera. 2007. Ten years of sustainability evaluations using de MESMIS framework: Lessons learned from its application in 28 Latin America case studies. International Journal of Sustainable Development \& World Ecology. 14 (4): 345-361.

Universidad Autónoma Benito Juárez de Oaxaca (UABJO). 2014. Plan municipal de desarrollo de la Heroica Ciudad de Juchitán de Zaragoza 2014-2016.

Valdez-Vazquez, I., C. del R. Sánchez G. \& A.E. Escalante. 2017. Proposal for a sustainability evaluation framework for bioenergy production systems using the MESMIS methodology. Renewable and Sustainable Energy Reviews. 68 (2017): 360-369.

World Commission on Environment and Development (WCED). 1987. Our common future. Oxford Univ. Press, Oxford.

Zagoya, M.J. 2015. Sistema tradicional utilizado en la producción de maíz en La Sierra Nevada de Puebla, México. Revista Caribeña de Ciencias Sociales 2015 (01):1-6 